\title{
Bioinformatics analysis of esophageal cancer unveils an integrated mRNA-IncRNA signature for predicting prognosis
}

\author{
TIAN LAN ${ }^{1,2}$, ZUNQIANG XIAO $^{2}$, HUA LUO $^{1}$, KUNLUN SU $^{1}$, \\ OUOU YANG ${ }^{1}$, CHENGNI ZHAN $^{1}$ and YUNYAN LU ${ }^{3}$ \\ ${ }^{1}$ Department of Breast Surgery, Hangzhou Hospital of Traditional Chinese Medicine, Hangzhou, Zhejiang 310007; \\ ${ }^{2}$ The Second Clinical Medical College, Zhejiang Chinese Medical University, Hangzhou, Zhejiang 310053; \\ ${ }^{3}$ Department of Cardiology, The First People's Hospital of Xiaoshan, Hangzhou, Zhejiang 311201, P.R. China
}

Received June 19, 2019; Accepted November 8, 2019

DOI: $10.3892 / 01.2019 .11208$

\begin{abstract}
Esophageal cancer (ESCA) carries a poor prognosis among gastrointestinal malignancies. The present study developed a signature based on mRNAs and long non-coding RNAs (lncRNAs) to predict prognosis in ESCA by using The Cancer Genome Atlas database. By using least absolute shrinkage and selection operator penalized regression, a set of RNAs (three mRNAs and two lncRNAs) was identified and used to build a risk score system of ESCA prognosis, which was used to stratify patients having considerable diverse survival in the training set [hazard ratio (HR), 3.932; 95\% CI, 1.555-9.944; $\mathrm{P}<0.002$ ] into high- and low-risk groups. The authentication of the results was achieved through the test set (HR, 3.150; 95\% CI, 1.113-8.918; $\mathrm{P}<0.02)$ and the entire set $(\mathrm{HR}, 3.181 ; 95 \% \mathrm{CI}, 1.686-6.006$; $\mathrm{P}<0.0002)$. The results from multivariate Cox proportional hazard regression analysis in the entire set suggested that the prognostic significance of this signature may be independent of patients' clinicopathological characteristics. Furthermore, this signature was associated with several molecular signaling pathways of cancer according to Gene Set Enrichment Analysis. In addition, a nomogram was built and the risk score and TNM stage were integrated to estimate the 1- and 3-year overall survival rates. The results from the present study demonstrated that the integrated mRNA-lncRNA signature may be considered as a novel biomarker for the prognosis of ESCA.
\end{abstract}

\section{Introduction}

Esophageal cancer (ESCA) ranks sixth in terms of cancer-associated mortality worldwide (1). In 2012, 455,000 patients were diagnosed with ESCA worldwide,

Correspondence to: Dr Yunyan Lu, Department of Cardiology, The First People's Hospital of Xiaoshan, 1099 Shixin South Road, Hangzhou, Zhejiang 311201, P.R. China

E-mail: 1yy10214@126.com

Key words: esophageal cancer, prognostic signature, The Cancer Genome Atlas, least absolute shrinkage and selection operator translating to an incidence rate of 5.9 per 100,000 (2). The 5 -year survival rate of patients with ESCA is only $18 \%$, because ESCA typically exhibits no symptoms and is therefore diagnosed at an advanced clinical stage (3). At present, the top predictive characteristic of ESCA prognosis is TNM staging (4). Although TNM stage is useful, it varies among patients with the same cancer stage (5). Furthermore, clinicopathological characteristics are molecular comprehensive reflections, including proteins and genes. Patients with ESCA with homogeneous clinical stage may be further classified according to various molecular patterns, like a 6-microRNA signature reported by Lan et al (6). It is therefore crucial to determine novel biomarkers for identifying patients with high risk of mortality.

Long non-coding RNA (lncRNA) is defined as an RNA transcript that is not translated into proteins and of $>200$ nucleotides in length (7). Conversely, with protein coding genes, the gene expression levels of reported IncRNAs are much lower (8). Furthermore, the role of IncRNAs is crucial in tumor growth, progression and treatment response (9), and most mRNAs serve critical roles in fundamental cellular processes. Numerous studies reported that integrated analysis of mRNAs and lncRNAs can contribute to the prognosis evaluation of breast and hepatocellular carcinoma $(10,11)$. However, only a few studies have focused on the integrated assessment of mRNAs and lncRNAs in ESCA.

The present study aimed to analyze the gene expression profile of ESCA samples from The Cancer Genome Atlas (TCGA) database, and a signature was constructed by integrating mRNA and lncRNA expression for the prediction of ESCA prognosis.

\section{Materials and methods}

Data source and preprocessing. The TCGA database (accessed January 2019; https://portal.gdc.cancer.gov/) provided the RNA sequencing (RNA-seq) data and clinicopathological characteristics of patients with ESCA collected between December 2011 and December 2013 (12). The inclusion criteria were as follows: i) Patients with ESCA, RNA-seq data and clinicopathological information; and ii) patients with ESCA with at least 30 days follow-up. A total of 117 samples were selected 
in the present study, including 106 tumor tissues and 11 normal tissues (Table SI). Only RNAs with transcript per million value $>0.1$ in $\geq 50 \%$ of ESCA samples were enrolled for further investigation. A total of 16,368 mRNAs and 7,347 lncRNAs were annotated according to GENCODE datasets (www. gencodegenes.org). The differentially expressed (DE) mRNAs and IncRNAs in tumor tissues compared with normal tissues were analyzed using the 'limma' package (version 3.36.5; http://bioconductor.org/packages/release/bioc/html/limma.

html) in R (version 3.5.2; https://www.r-project.org/). The results were visualized by volcano plot. Due to the gap between the count of mRNAs and lncRNAs, different thresholds were set for identifying DE mRNAs and lncRNAs. DEmRNAs with $\mathrm{P}<0.05$ and $\mid \log$ fold change $(\mathrm{FC}) \mid>2.0$, and DElncRNAs with $\mathrm{P}<0.05$ and $\mid \log \mathrm{FCl}>1.0$ were considered as significant. The research process scheme is presented in Fig. 1. Since the TCGA database information is publicly available, no ethical approval was required.

Construction and validation of the mRNA-lncRNA signature. The prognostic values of mRNAs and lncRNAs were determined using univariate Cox proportional hazards regression analysis. According to previous studies, $\mathrm{P}<0.1$ was considered to indicate a statistically significant difference $(6,13,14)$. Subsequently, a total of 106 tumor samples were randomly divided into two sets: A training set $(n=53)$ and a test set $(n=53$; Table I).

The least absolute shrinkage and selection operator (LASSO) method, which is appropriate for high-dimensional data analysis (15), can select an optimal group of genes lacking collinearity through penalty imposing and most regression coefficients shrinking to zero. LASSO was therefore used in the training set to determine and confirm the selected mRNAs and lncRNAs. The prognostic mRNA-lncRNA signature risk score in patients was calculated using each prognostic RNA expression level and its associated coefficient. The formula used was as follow: Risk score $=\beta 1 \times$ gene $1+\beta 2 \times$ gene $2+\ldots+$ $\beta \mathrm{n} x$ gene $\mathrm{n}$, where $\beta$ indicates the coefficient of each gene and gene indicates the expressed gene value.

Based on the median risk score cut-off (2.541), patients were divided into a high-risk group and a low-risk group. Kaplan-Meier (KM) and log-rank methods were used to test the difference in the two groups by using the 'survival' R package (version 2.43; https://cran.r-project.org/web/packages/survival/index.html). To validate the signature sensitivity and precision, the 'timeROC' $\mathrm{R}$ package (version 0.3; https://cran.r-project.org/web/packages/timeROC/index.html) was used to calculate the area under the receiver operating characteristic (ROC) curves. This signature was further validated in the test set and the entire set. Subsequently, stratified analysis based on the clinicopathological characteristics of patients with ESCA was carried out in the entire set.

Development of the nomogram. The prognostic significance of the signature was evaluated with clinicopathologic characteristics, including age, sex, height, weight, pathology, alcohol consumption history, Barrett's disease and TNM stage, by univariate and multivariate Cox proportional hazard regressions analysis. In order to provide clinicians with a quantitative tool to predict the individual probability of survival time,

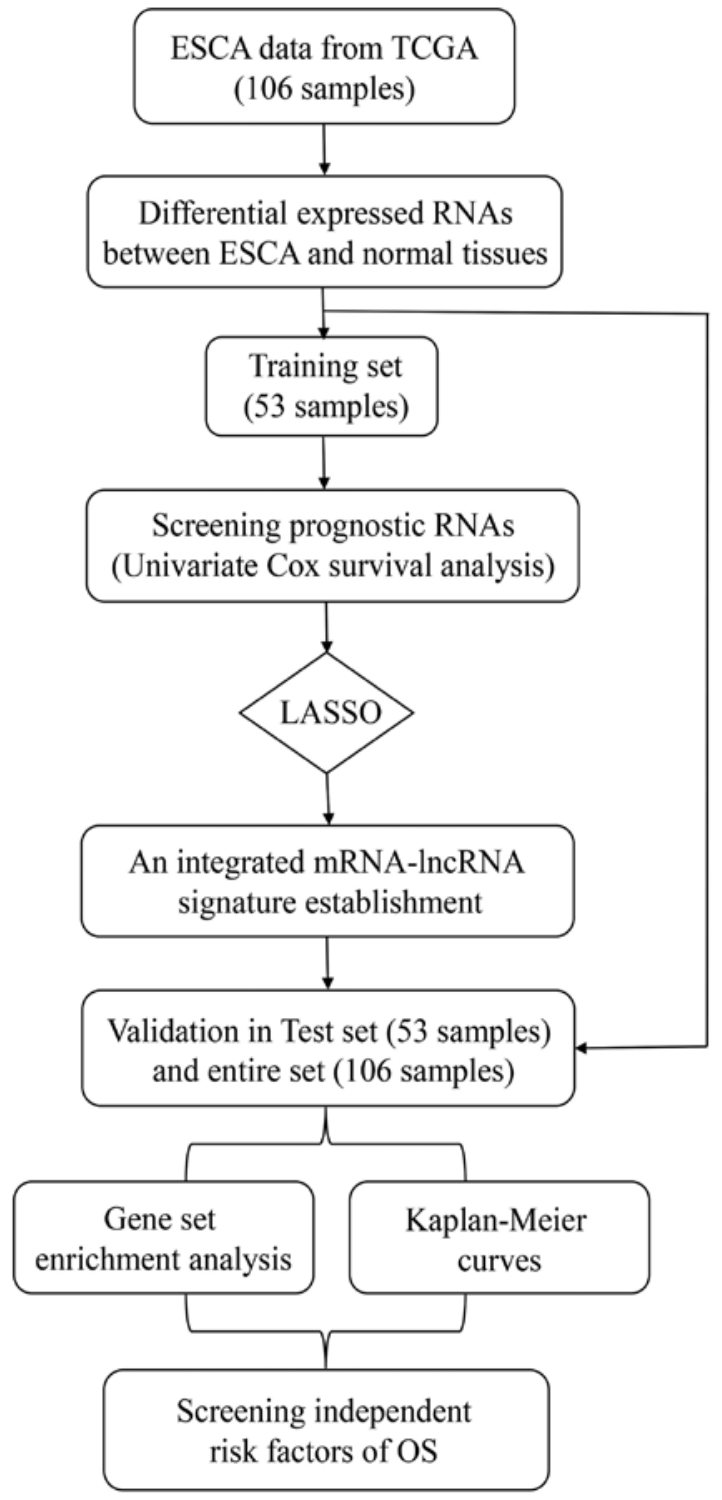

Figure 1. Overview of the analytic workflow of the present study. ESCA, esophageal carcinoma; LASSO, least absolute shrinkage and selection operator; lncRNA, long non-coding RNA; OS, overall survival; TCGA, The Cancer Genome Atlas.

the $\mathrm{R}$ package 'rms' (version 5.1-3; https:/cran.r-project. org/web/packages/rms/) was used to build the nomogram associated with the variables, including a mRNA-lncRNA signature and TNM stage, derived from the previous analysis. Furthermore, the nomogram predictive performance was calculated using concordance index and bootstrapping validation calibration (1,000 bootstrap resamples) to reduce the potential of overfitting.

mRNA-lncRNA signature function prediction. To study the potential biological mechanism between low- and high-risk groups, gene set enrichment analysis (GSEA; http://software.broadinstitute.org/gsea) was performed. The BioCarta (c2.cp.biocarta.v6.2.symbols.gmt) and Reactome (c2.cp.reactome.v6.2.symbols.gmt) datasets (http://software. broadinstitute.org/gsea/msigdb/collections.jsp) were selected as the reference gene sets. A gene with $\mathrm{P}<0.05$ and enrichment score $>0.5$ was considered as significantly enriched. 
Table I. Characteristics of patients in the training and test set.

\begin{tabular}{|c|c|c|c|}
\hline Variables & Training set, n (\%) & Test set, n (\%) & P-value \\
\hline Number & 53 & 53 & \\
\hline Age, years $($ mean \pm SD) & $65.00 \pm 12.41$ & $64.06 \pm 12.34$ & 0.695 \\
\hline \multicolumn{4}{|l|}{ Sex } \\
\hline Female & $11(20.8)$ & $8(15.1)$ & 0.613 \\
\hline Male & $42(79.2)$ & $45(84.9)$ & \\
\hline \multicolumn{4}{|l|}{ Height, cm } \\
\hline$<175$ & $28(57.1)$ & $29(59.2)$ & $>0.999$ \\
\hline$>175$ & $21(42.9)$ & $20(40.8)$ & \\
\hline No value & 4 & 4 & \\
\hline \multicolumn{4}{|l|}{ Weight, kg } \\
\hline$<80$ & $32(60.4)$ & $27(51.9)$ & 0.434 \\
\hline$>80$ & $21(39.6)$ & $25(48.1)$ & \\
\hline No value & 0 & 1 & \\
\hline \multicolumn{4}{|l|}{ Ethnicity } \\
\hline Non-white & $3(7.1)$ & $7(15.2)$ & 0.320 \\
\hline White & $39(92.9)$ & $39(84.8)$ & \\
\hline No value & 11 & 7 & \\
\hline \multicolumn{4}{|l|}{ Pathology } \\
\hline Adenocarcinoma & $40(75.5)$ & $35(66.0)$ & 0.393 \\
\hline Squamous & $13(24.5)$ & $18(34.0)$ & \\
\hline \multicolumn{4}{|l|}{ Alcohol history } \\
\hline No & $17(32.7)$ & $15(28.3)$ & 0.675 \\
\hline Yes & $35(67.3)$ & $38(71.7)$ & \\
\hline No value & 1 & 0 & \\
\hline \multicolumn{4}{|l|}{ Barrett's disease } \\
\hline No & $34(69.4)$ & $40(81.6)$ & 0.240 \\
\hline Yes & 15 (30.6) & $9(18.4)$ & \\
\hline No value & 4 & 4 & \\
\hline \multicolumn{4}{|l|}{$\mathrm{T}$} \\
\hline $1+2$ & $23(44.2)$ & $22(42.3)$ & $>0.999$ \\
\hline $3+4$ & $29(55.8)$ & $30(57.7)$ & \\
\hline No value & 1 & 1 & \\
\hline \multicolumn{4}{|l|}{$\mathrm{N}$} \\
\hline 0 & $16(33.3)$ & $16(33.3)$ & $>0.999$ \\
\hline $1+3$ & $32(66.7)$ & $32(66.7)$ & \\
\hline No value & 5 & 5 & \\
\hline \multicolumn{4}{|l|}{ M } \\
\hline 0 & $42(87.5)$ & $36(83.7)$ & 0.766 \\
\hline 1 & $6(12.5)$ & $7(16.3)$ & \\
\hline No value & 5 & 10 & \\
\hline \multicolumn{4}{|l|}{ Stage } \\
\hline $\mathrm{I}+\mathrm{II}$ & $29(54.7)$ & $24(48.0)$ & 0.557 \\
\hline III+IV & $24(45.3)$ & $26(52.0)$ & \\
\hline No value & 0 & 3 & \\
\hline \multicolumn{4}{|l|}{ Survival status } \\
\hline Alive & $27(50.9)$ & $33(62.3)$ & 0.327 \\
\hline Dead & $26(49.1)$ & $20(37.7)$ & \\
\hline Survival time, years (mean \pm SD) & $1.47 \pm 1.35$ & $1.34 \pm 1.16$ & 0.606 \\
\hline
\end{tabular}


Table II. Univariate and multivariate Cox regression analysis of risk score and clinical variables.

\begin{tabular}{|c|c|c|c|c|c|c|}
\hline \multirow[b]{2}{*}{ Characteristics } & \multicolumn{3}{|c|}{ Univariate analysis } & \multicolumn{3}{|c|}{ Multivariate analysis } \\
\hline & HR & $95 \% \mathrm{CI}$ & P-value & HR & $95 \% \mathrm{CI}$ & P-value \\
\hline Risk score & 2.50 & $1.55-4.06$ & $<0.001$ & 2.41 & $1.47-3.96$ & $<0.001$ \\
\hline Age ( $\geq 60$ years vs. $<60$ years) & 0.68 & $0.36-1.29$ & 0.238 & & & \\
\hline Sex (male vs. female) & 1.70 & $0.52-5.54$ & 0.382 & & & \\
\hline Height $(\geq 175 \mathrm{~cm}$ vs. $<175 \mathrm{~cm})$ & 1.15 & $0.60-2.19$ & 0.681 & & & \\
\hline Weight $(\geq 85 \mathrm{~kg}$ vs. $<85 \mathrm{~kg}$ ) & 0.79 & $0.41-1.51$ & 0.471 & & & \\
\hline Pathology (squamous vs. adenocarcinoma) & 1.04 & $0.51-2.12$ & 0.903 & & & \\
\hline Alcohol consumption (yes vs. no) & 0.57 & $0.30-1.08$ & 0.083 & & & \\
\hline Barrett's disease (yes vs. no) & 1.46 & $0.74-2.88$ & 0.271 & & & \\
\hline Stage (III+IV vs. I+II) & 2.09 & $1.29-3.41$ & 0.003 & 1.98 & $1.20-3.27$ & 0.007 \\
\hline
\end{tabular}

HR, hazard ratio.
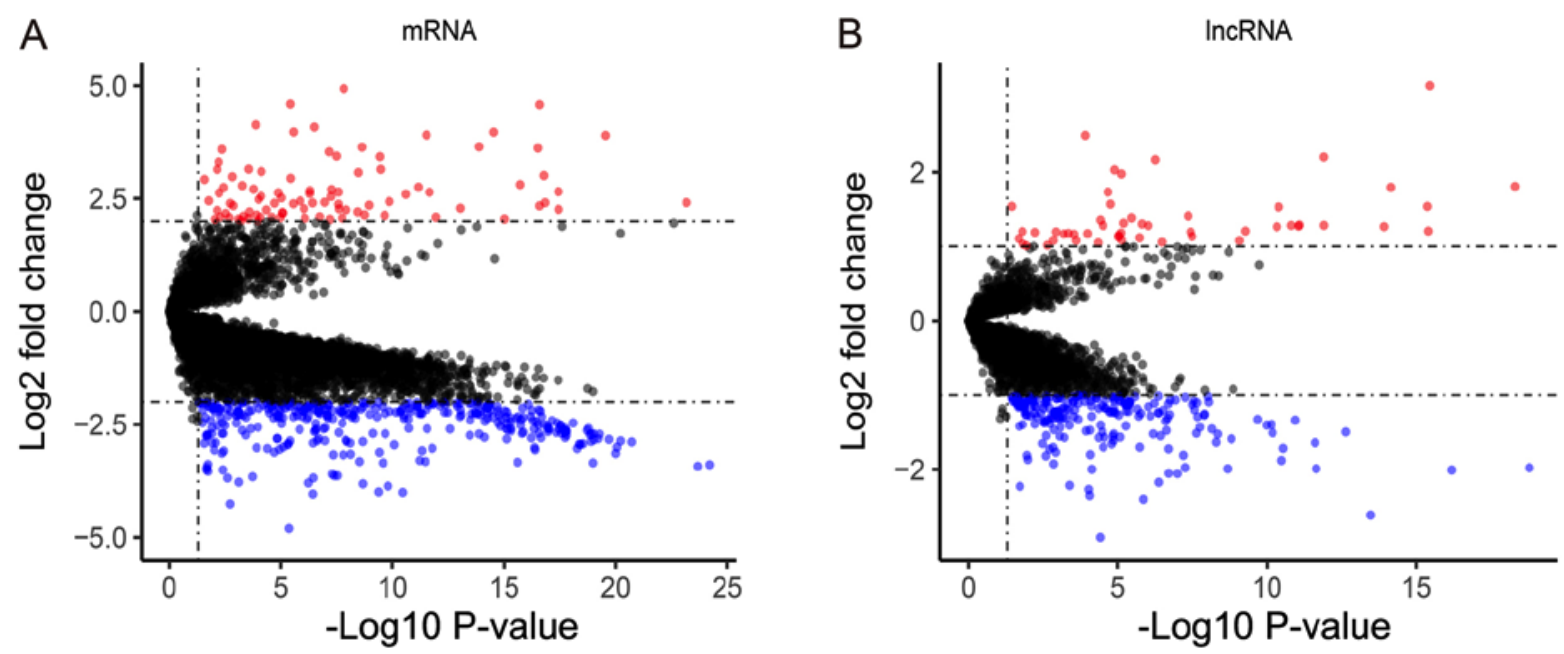

Figure 2. Volcano plot of differentially expressed (A) mRNAs and (B) lncRNAs between esophageal carcinoma and non-tumor tissues. The red points indicate upregulated genes and the blue points represent downregulated genes. IncRNA, long non-coding RNA.
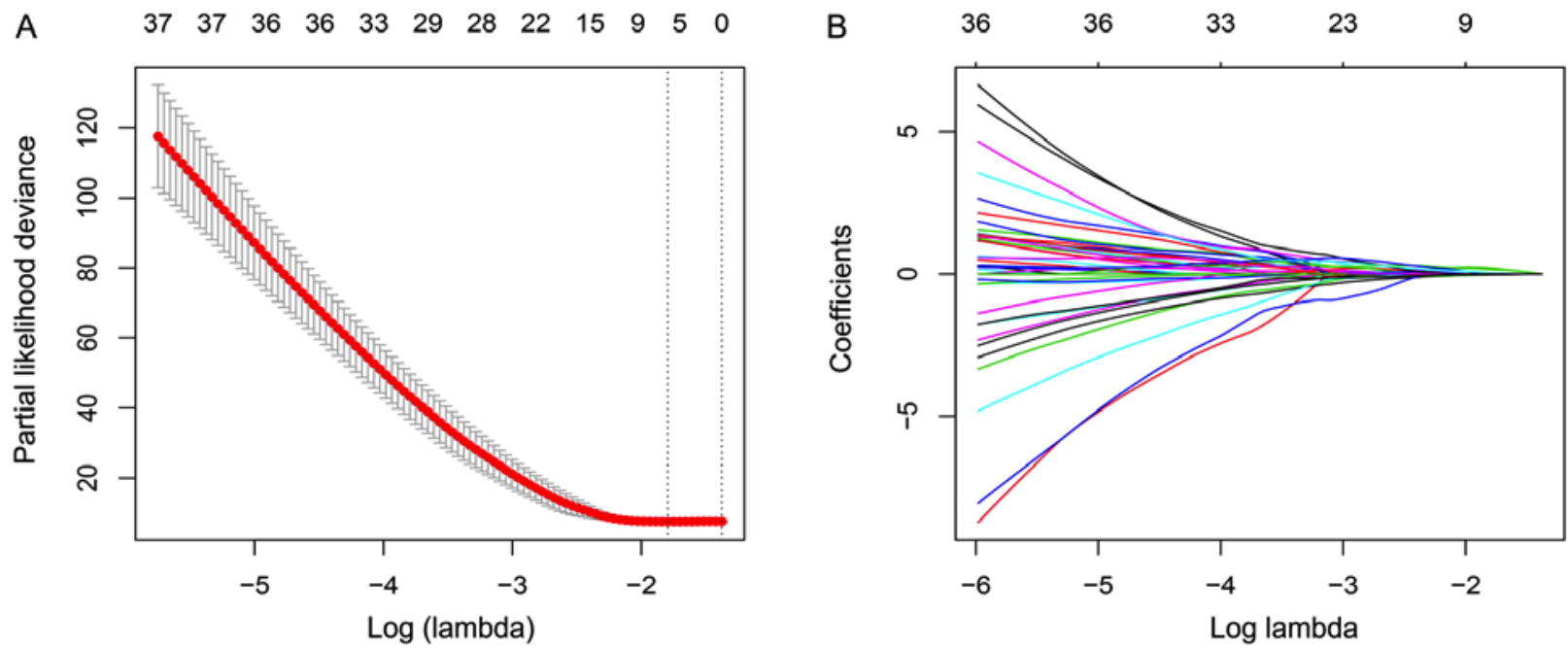

Figure 3. Texture feature selection using LASSO regression model. (A) Lambda selection in the LASSO model using 10-fold cross-validation. Dotted vertical lines on the left and right represent, respectively, the value with the minimum error and the largest lambda value where the deviance is within one standard error of the minimum. (B) LASSO coefficient profiles of the differentially expressed genes associated with the overall survival of patients with esophageal carcinoma. LASSO, least absolute shrinkage and selection operator. 


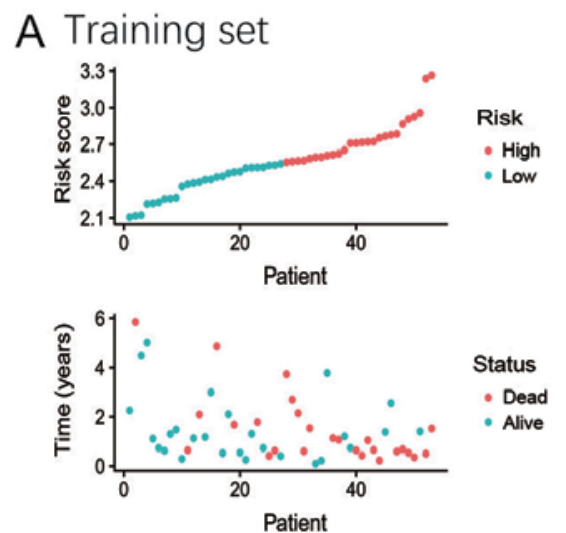

\section{B Test set}
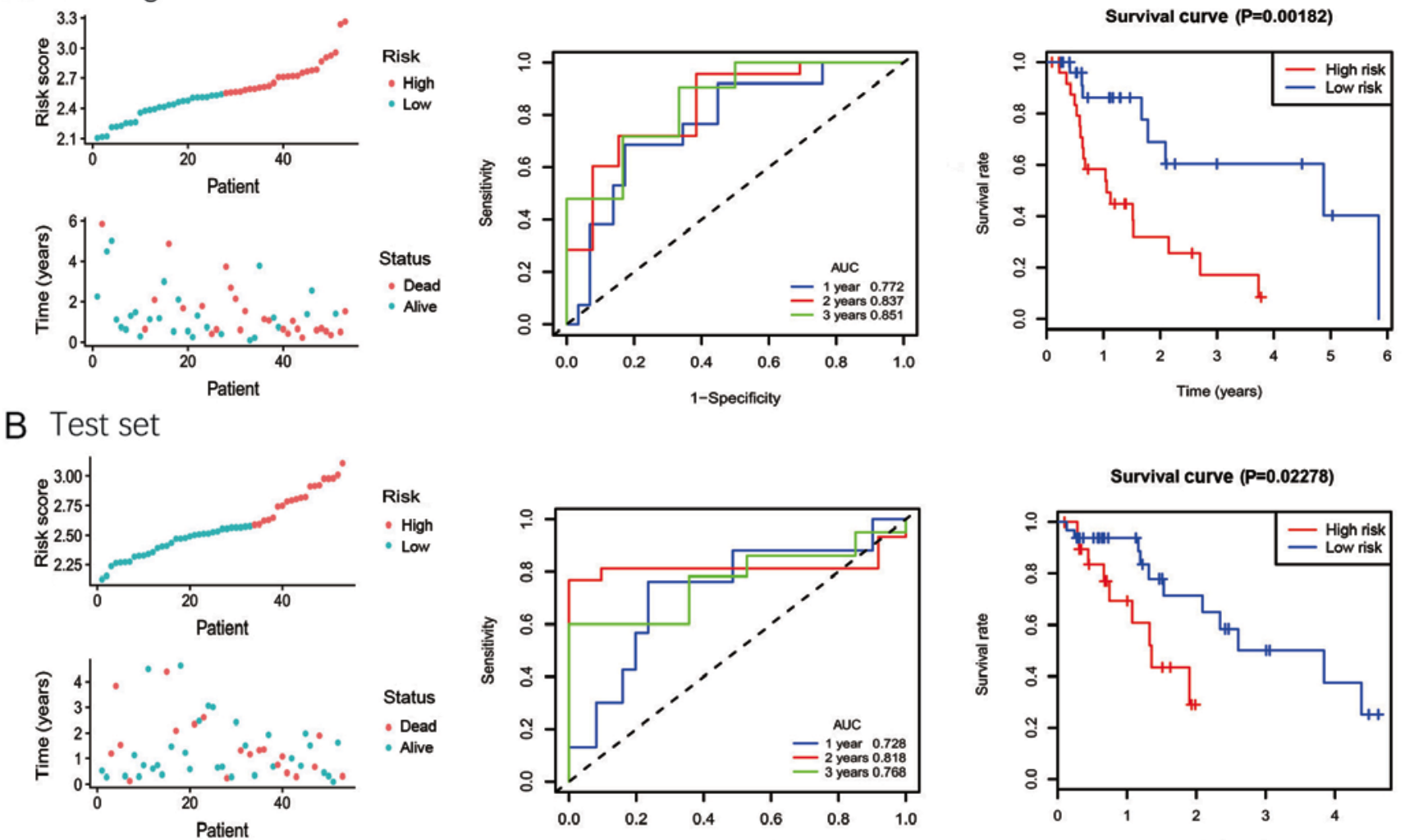

\section{Entire set}
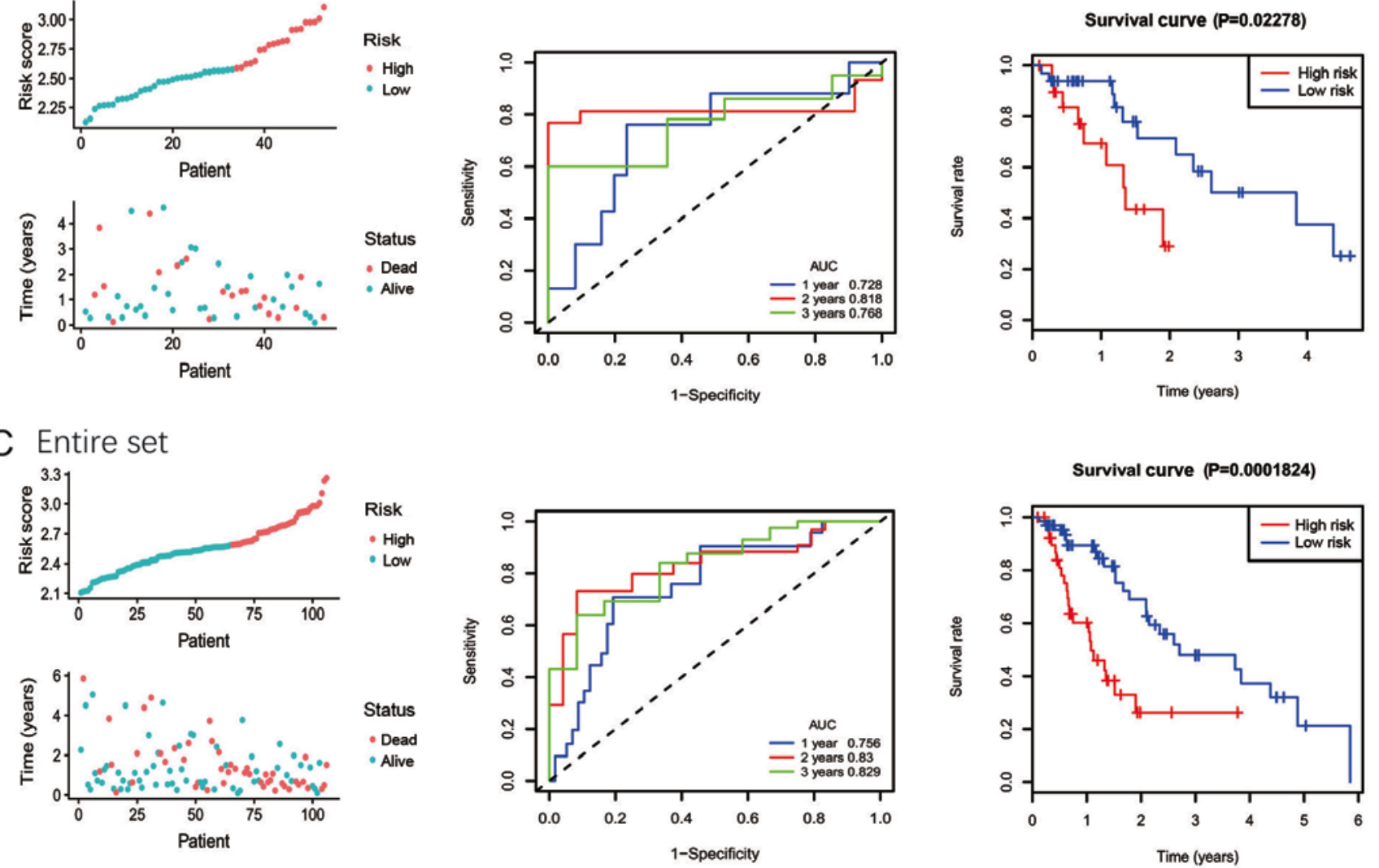

Figure 4. Construction and validation of the signature. Distribution of risk group, risk score and survival status (left panel), time dependent receiver operating characteristic curves at 1,2 and 3 years (middle panel) and Kaplan-Meier survival analysis between high- and low-risk groups (right panel) in the (A) training, (B) test and (C) entire sets. AUC, area under the curve.

\section{Results}

DEmRNAs and DElncRNAs in patients with ESCA. A total of 440 mRNAs were identified as significantly different between tumor and normal tissues, of which 93 mRNAs were upregulated and 347 were downregulated (Fig. 2A). In addition, 263 DElncRNAs (51 upregulated; 212 downregulated) were identified and selected for further analysis (Fig. 2B).

Signature development in the training set. A total of 52 DEmRNAs and 38 DElncRNAs were identified in the training set as being associated with prognosis following univariate Cox proportional hazards regression analysis (Table SII). Furthermore, using the LASSO method, three
mRNAs, PCNA, TNS4, SLC26A9, and two lncRNAs, ZFAS1, AC104041.1, were identified (Fig. 3B). An appropriate value of lambda was set as 0.166 using cross validation (Fig. 3A). The risk score calculated from the five expressed genes weighted by their coefficients was set as below: Risk score $=(0.209095$ $\mathrm{x}$ expression of PCNA $)+(-0.023333 \mathrm{x}$ expression of TNS4) + $(-0.025136 \times$ expression of SLC26A9) + (0.160318 x expression of ZFAS1 $)+(0.035372 \mathrm{x}$ expression of AC104041.1).

According to the median risk score, patients were divided into high- and low-risk groups. The risk scores, survival time distributions and patients' status in the training set are presented in Fig. 4A (left panel). The results from KM survival analysis demonstrated that the two groups had significantly different outcomes (Fig. 4A; right panel). In addition, 1-, 
A

Points

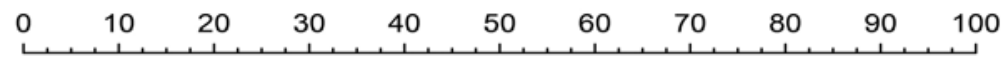

Risk score

Stage

Total points

1-year survival probability

3-year survival probability

$\mathrm{B}$

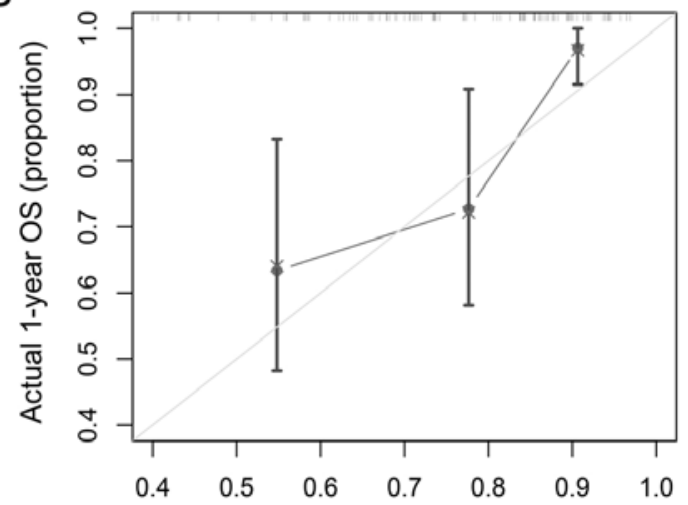

Nomogram-predicted probability of 1 -year OS

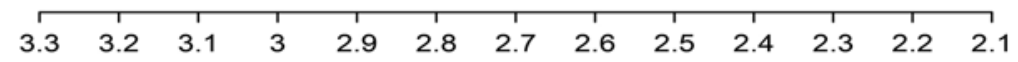

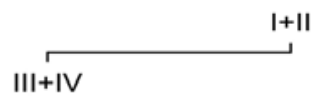

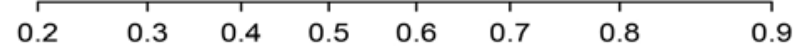

\begin{tabular}{lllllll}
\hline 0.1 & 0.2 & 0.3 & 0.4 & 0.5 & 0.6 & 0.7
\end{tabular}

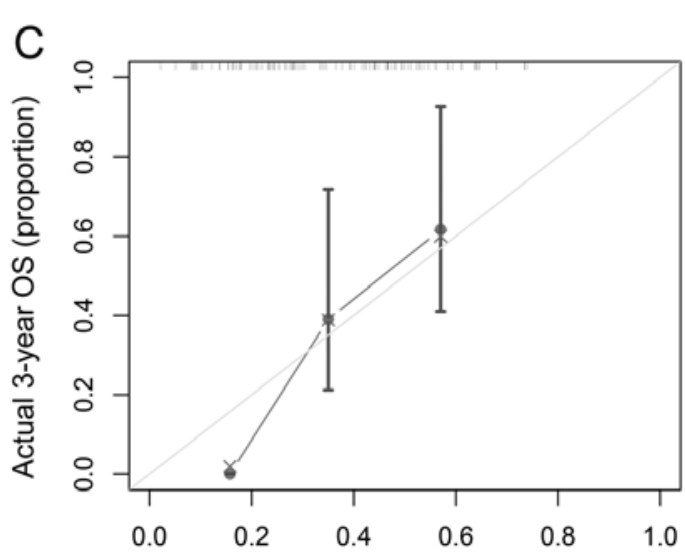

Nomogram-predicted probability of 3-year OS

Figure 5. Nomogram construction and validation. (A) Nomogram developed with risk score and TNM stage for 1- and 3-year survival probability in the primary cohort. Calibration curves of nomograms in terms of agreement between predicted and observed (B) 1-and (C) 3 -year outcomes in the primary cohort. OS, overall survival.

2- and 3-year time-dependent ROC analyses were performed to evaluate the mRNA-lncRNA signature prognostic sensitivity and specificity (Fig. 4A; middle panel). The AUCs of the mRNA-lncRNA signature were $0.772,0.837$ and 0.851 at $1-$, 2- and 3-year survival times, respectively, suggesting that this signature may have a high prognostic accuracy.

Validation of the signature in the test and entire sets. By using the established cut-off point, patients were assigned to a lowor high-risk group in the test and entire sets. The patients' risk score distribution and survival status were ranked by the risk scores in the test set (Fig. 4B, left panel) and the entire set (Fig. 4C, left panel). The results from KM analysis for overall survival (OS) indicated that patients with low risk may exhibit improved clinical outcomes compared with patients with high risk (Fig. 4B and C, right panel). To confirm the accuracy of the signature, the areas under ROC curves were calculated, and the results were $0.728,0.818$ and 0.768 at 1-, 2- and 3-year survival times in the test set (Fig. 4B, middle panel), respectively, and $0.756,0.830,0.829$ at 1-, 2- and 3-year survival times in the entire set (Fig. 4C, middle panel), respectively.
Stratified analysis and independence analysis in the entire set. The results from subgroup analysis based on age, sex, height, weight, ethnicity, alcohol consumption history, Barrett's disease and TNM stage suggested that patients with high-risk of mortality may present with poor clinical outcomes. The results were all significant, except for women, non-white, no alcohol consumption history, tumor size I+II and stage I+II (Fig. S1). Furthermore, the mRNA-lncRNA signature was considered as an independent prognostic factor in univariate and multivariate Cox regression analyses in the entire set following adjustment for various clinicopathological characteristics [hazard ratio (HR), 2.5, 95\% confidence interval (CI), 1.55-4.06, $\mathrm{P}<0.001$ in the univariate $\mathrm{Cox}$ regression analysis; $\mathrm{HR}, 2.41,95 \% \mathrm{CI}, 1.47-3.96, \mathrm{P}<0.001$ in the multivariate Cox regression analysis; Table II].

Construction of a novel nomogram for predicting prognosis in ESCA. Risk score and TNM stage derived from the previous analyses were developed and presented as a nomogram (Fig. 5A). A high score of the nomogram indicated a high probability of 1- and 3-year survival. In the primary cohort, 
A

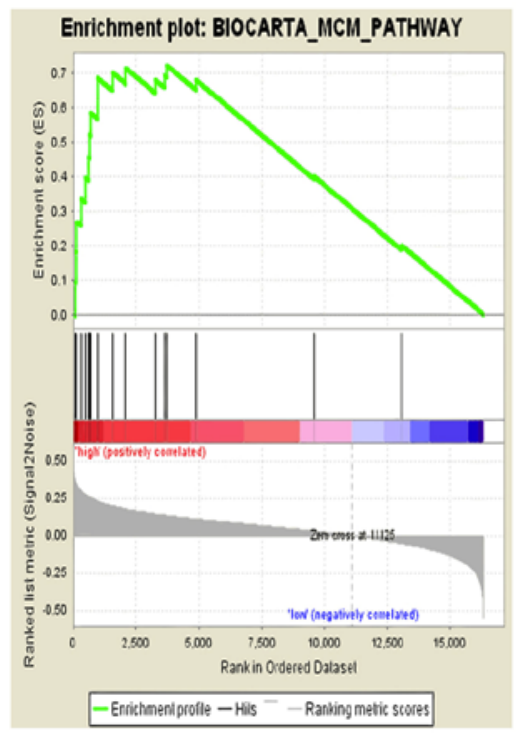

B
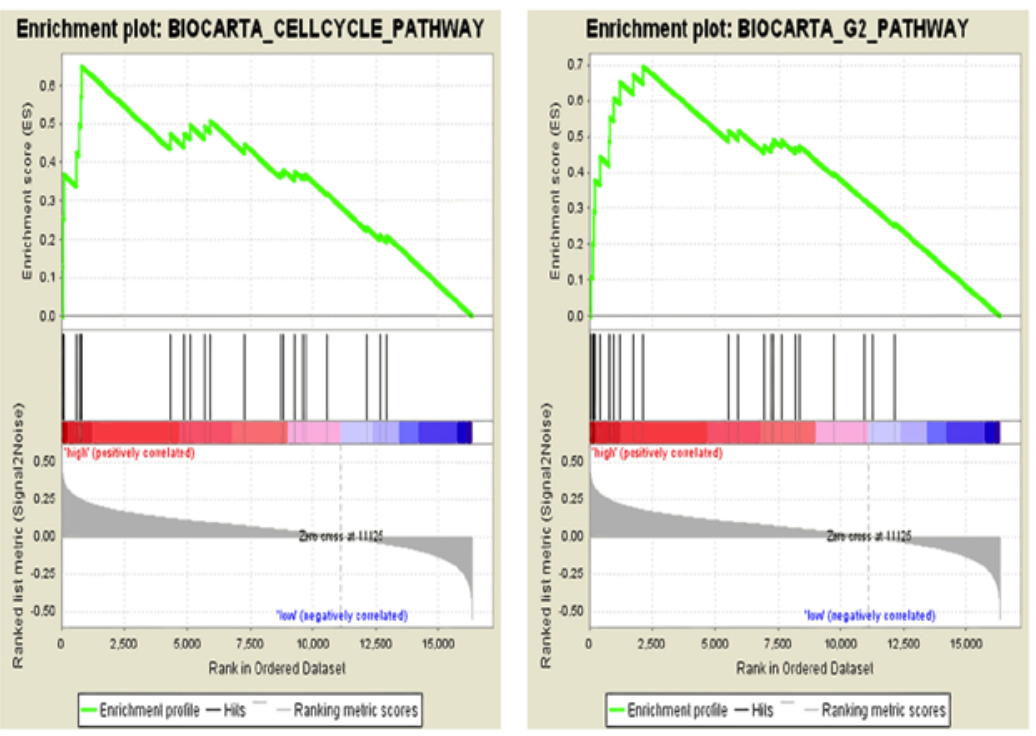

\section{Reactome}

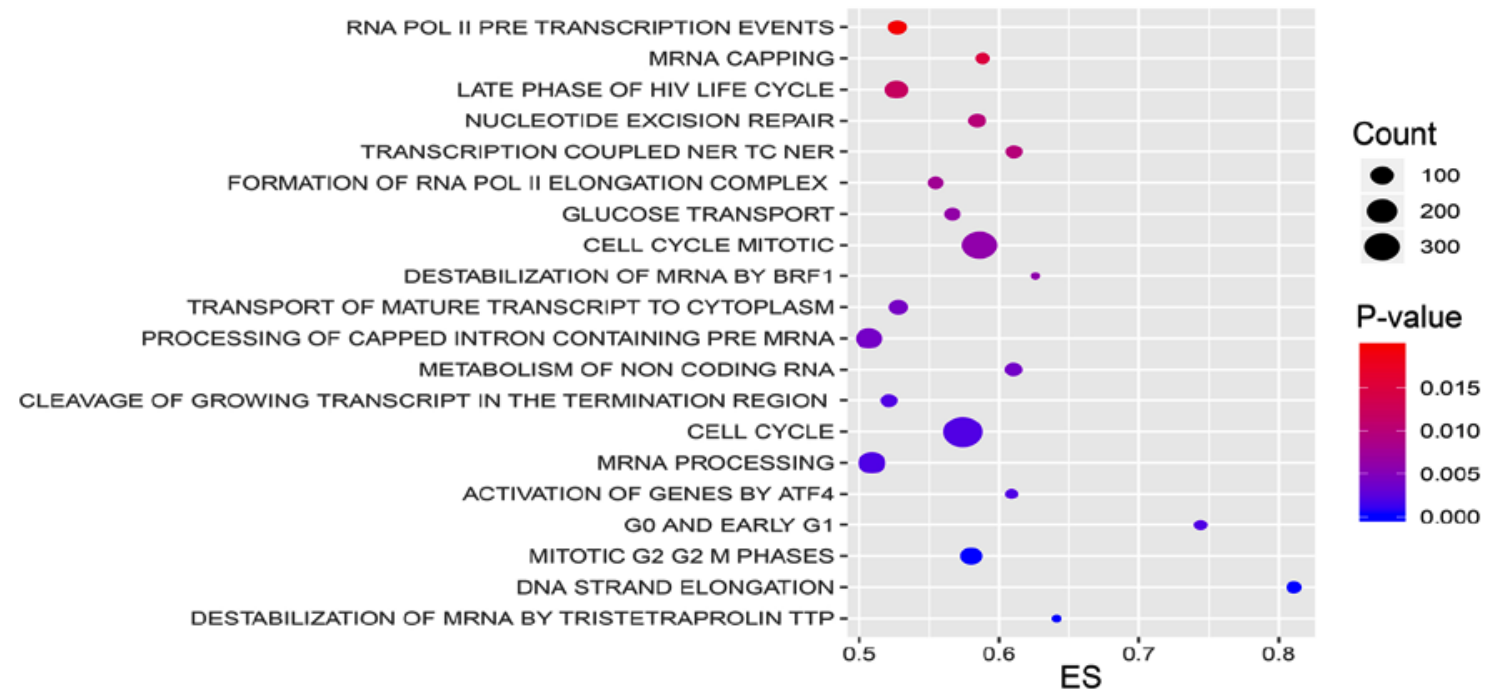

Figure 6. Gene enrichment analysis of the signature. (A) Pathways 'MCM', 'cell cycle' and 'G2' were significantly enriched using the gene set of 'C2. cp.biocarta.v6.2.symbols.gmt'. (B) Results from gene set enrichment analysis delineated signalling pathway associated with the mRNA-lncRNA signature using the gene set of 'c2.cp.reactome.v6.2.symbols.gmt'. Node size represents the number of genes enriched. Each node was colored according to the P-value. MCM, minichromosome maintenance.

the nomogram C-index was 0.717 (95\% CI, 0.543-0.891). The established nomogram calibration curve for the probability of survival outcome indicated good agreement between observation and prediction (Fig. 5B and C).

Functional analysis of the mRNA-IncRNA signature. In order to determine the possible signature mechanism, BioCarta and Reactome pathway enrichment analysis was performed using GSEA. The results demonstrated that compared with genes in the low-risk group, genes in the high-risk group were significantly enriched with numerous biological processes, in particular cell cycle signaling pathway, minichromosome maintenance pathway and mRNA capping and processing pathway (Fig. 6).

\section{Discussion}

Clinicopathological characteristics of patients with ESCA, including TNM stage, have been widely used to adapt individual treatment and predict prognosis for patients. However, due to the heterogeneity of ESCA, it is difficult to precisely predict prognosis by using clinicopathological characteristics (16). Therefore, application of gene-based biomarkers represents a promising alternative to predict prognosis.

Numerous studies have identified molecular markers for prognosis in ESCA. Peters et al (17) generated a 4-mRNA prognostic signature for patients with esophageal adenocarcinoma. Recently, a reliable 6-immunohistochemical-based signature has been determined for patients with esophageal squamous cell cancer (18). Furthermore, a 3-IncRNA signature associated with OS has been built for patients with esophageal squamous cell carcinoma (19). Fan et al (20) developed a novel 8-IncRNA signature for the prediction of prognosis in patients with ESCA. Additionally, a previous study reported that the dysregulated expression of certain micro (mi)RNAs (hsa-mir-425, hsa-let-7b, hsa-mir-23a, hsa-mir-3074, hsa-mir-424 and hsa-mir-505) could be used for 
the prognosis of esophageal adenocarcinoma (6). To the best of our knowledge, previous studies have only focused on mRNAs, lncRNAs or miRNAs, without investigating multi-RNA-based data. It is therefore crucial to assess whether multi-RNA-based signatures could predict prognosis in ESCA.

In the present study, a mRNA-IncRNA prognostic signature was established by conducting LASSO Cox regression model analysis. Based on the risk score of the signature, patients were divided into high- and low-risk groups. Patients in the low-risk group exhibited improved clinical outcomes. Furthermore, the mRNA-lncRNA signature was independent of the clinicopathological characteristics in univariate and multivariate Cox regression analyses.

The prognostic signature for patients with ESCA contained five genes, three mRNAs, PCNA, TNS4, SLC26A9, and two lncRNAs, ZFAS1, AC104041.1.

Among them, the genes proliferating cell nuclear antigen (PCNA), ZNFX1 antisense RNA 1 (ZFAS1) and AC10401.1 were negatively associated with OS, whereas the two other genes tensin 4 (TNS4) and solute carrier family 26 member 9 (SLC26A9) were associated with an improved survival outcome. PCNA has been identified as a proliferating cell nuclear antigen that can serve a role in DNA replication and repair (21). Furthermore, previous studies have identified PCNA as a cell growth marker and a predictive indicator of various types of cancer, including colorectal cancer, gastric carcinoma and parotid gland cancer (22-24). In addition, it has been reported that upregulated PCNA is associated with poor survival in patients with esophagus squamous cell cancer (25), which is consistent with the results from our previous study on ESCA. Furthermore, Kimos et al (26) identified PCNA as a biomarker of esophageal neoplastic progression, suggesting that PCNA could be a target for ESCA management. TNS4, which is identified as a $\mathrm{COOH}$-terminus tensin-like molecule, has been reported to be a cell adhesion factor that is associated with cancer cell mobility and migration (27). Previous studies have demonstrated that TNS4 can promote cancer cell migration, and can be considered as an oncogene in hepatocellular cancer (28), pancreatic cancer (29) and colon carcinoma (30). Furthermore, none or lowly expressed TNS4 has been reported in kidney and prostate cancer $(31,32)$. These findings suggest the biological functions of TNS4 are dependent on the type of cancer. In the present study, reduced expression of TNS4 in ESCA samples was associated with short survival in patients. Regarding ZFAS1, its overexpression has been reported to be associated with metastasis and poor prognosis in gastric cancer (33), colorectal cancer (34) and hepatocellular carcinoma (35), suggesting that ZFAS1 may be considered as a potential prognostic marker in cancer. Consistently, the present study demonstrated that ZFAS1 expression was inversely associated with the OS of patients with ESCA. Regarding SLC26A9 and AC104041.1, there was no correlative literature in cancer. The biological role of these genes in ESCA should be further investigated.

The present study presented some limitations. Firstly, only 106 ESCA samples were available in the TCGA dataset, hence the sub-group sample size was too small. Secondly, the identified signature should be further validated in an external dataset. Thirdly, in vitro and in vivo experiments should be performed in order to better understand the potential mechanism of this signature.
In conclusion, the present study established and validated a mRNA-lncRNA integrated signature for the prognosis of patients with ESCA by using a TCGA dataset. Although further investigation is required to confirm the importance of this signature, the present study provided valuable information regarding ESCA pathology and its clinical management.

\section{Acknowledgements}

Not applicable.

\section{Funding}

No funding was received.

\section{Availability of data and materials}

The datasets used and/or analyzed during the present study are available from the corresponding author on reasonable request.

\section{Authors' contributions}

TL and YL conceived, designed and performed the experiments. TL and YL analyzed the data, prepared figures and/or tables and wrote and revised the manuscript. ZX, KS and OY performed the experiments. HL and CZ analyzed the data. All authors read and approved the final version of the manuscript.

\section{Ethics approval and consent to participate}

Not applicable.

\section{Patient consent for publication}

Not applicable.

\section{Competing interests}

The authors declare that they have no competing interests.

\section{References}

1. Dy GW, Gore JL, Forouzanfar MH, Naghavi M and Fitzmaurice C: Global burden of urologic cancers, 1990-2013. Eur Urol 71: 437-446, 2017.

2. Arnold M, Soerjomataram I, Ferlay J and Forman D: Global incidence of oesophageal cancer by histological subtype in 2012 . Gut 64: 381-387, 2015.

3. Alsop BR and Sharma P: Esophageal cancer. Gastroenterol Clin North Am 45: 399-412, 2016.

4. Rice TW, Ishwaran H, Blackstone EH, Hofstetter WL, Kelsen DP and Apperson-Hansen C; Worldwide Esophageal Cancer Collaboration Investigators: Recommendations for clinical staging (cTNM) of cancer of the esophagus and esophagogastric junction for the 8th edition AJCC/UICC staging manuals. Dis Esophagus 29: 913-919, 2016.

5. Rice TW, Gress DM, Patil DT, Hofstetter WL, Kelsen DP and Blackstone EH: Cancer of the esophagus and esophagogastric junction-major changes in the American joint committee on cancer eighth edition cancer staging manual. CA Cancer J Clin 67: 304-317, 2017.

6. Lan T, Lu Y, Xiao Z, Xu H, He J, Hu Z and Mao W: A six-microRNA signature can better predict overall survival of patients with esophagus adenocarcinoma. PeerJ 7: e7353, 2019.

7. Wang $\mathrm{KC}$ and Chang HY: Molecular mechanisms of long noncoding RNAs. Mol Cell 43: 904-914, 2011. 
8. Yuan LY, Qin X, Li L, Zhou J, Zhou M, Li X, Xu Y, Wang XJ and Xing $H$ : The transcriptome profiles and methylation status revealed the potential cancer-related lncRNAs in patients with cervical cancer. J Cell Physiol 234: 9756-9763, 2019.

9. Huarte M: The emerging role of IncRNAs in cancer. Nat Med 21: $1253-1261,2015$

10. Jiang YZ, Liu YR, Xu XE, Jin X, Hu X, Yu KD and Shao ZM: Transcriptome Analysis of triple-negative breast cancer reveals an integrated mRNA-lncRNA signature with predictive and prognostic value. Cancer Res 76: 2105-2114, 2016.

11. Shi YM, Li YY, Lin JY, Zheng L, Zhu YM and Huang J: The discovery of a novel eight-mRNA-lncRNA signature predicting survival of hepatocellular carcinoma patients. J Cell Biochem. Nov. 28, 2018 (Epub ahead of print).

12. Cancer Genome Atlas Research Network; Analysis Working Group: Asan University; BC Cancer Agency; Brigham and Women's Hospital; Broad Institute; Brown University; Case Western Reserve University; Dana-Farber Cancer Institute; Duke University, et al: Integrated genomic characterization of oesophageal carcinoma. Nature 541: 169-175, 2017.

13. Gu J, Zhang X, Miao R, Ma X, Xiang X, Fu Y, Liu C, Niu W and Qu K: A three-long non-coding RNA-expression-based risk score system can better predict both overall and recurrence-free survival in patients with small hepatocellular carcinoma. Aging (Albany NY) 10: 1627-1639, 2018.

14. Lu JH, Zuo ZX, Wang W, Zhao Q, Qiu MZ, Luo HY, Chen ZH, Mo HY, Wang F, Yang DD, et al: A two-microRNA-based signature predicts first-line chemotherapy outcomes in advanced colorectal cancer patients. Cell Death Discov 4: 116, 2018.

15. Gao J, Kwan PW and Shi D: Sparse kernel learning with LASSO and Bayesian inference algorithm. Neural Netw 23: 257-264, 2010

16. Allison KH and Sledge GW: Heterogeneity and cancer. Oncology (Williston Park) 28: 772-778, 2014.

17. Peters CJ, Rees JR, Hardwick RH, Hardwick JS, Vowler SL, Ong CA, Zhang C, Save V, O'Donovan M, Rassl D, et al: A 4-gene signature predicts survival of patients with resected adenocarcinoma of the esophagus, junction, and gastric cardia Gastroenterology 139: 1995-2004 e15, 2010.

18. Meng J, Zhang J, Xiu Y, Jin Y, Xiang J, Nie Y, Fu S and Zhao K: Prognostic value of an immunohistochemical signature in patients with esophageal squamous cell carcinoma undergoing radical esophagectomy. Mol Oncol 12: 196-207, 2018.

19. Li J, Chen Z, Tian L, Zhou C, He MY, Gao Y, Wang S, Zhou F, Shi S, Feng X, et al: LncRNA profile study reveals a three-lncRNA signature associated with the survival of patients with oesophageal squamous cell carcinoma. Gut 63: 1700-1710, 2014

20. Fan Q and Liu B: Identification of a RNA-Seq based 8-long non-coding RNA signature predicting survival in esophageal cancer. Med Sci Monit 22: 5163-5172, 2016

21. Wang SC: PCNA: A silent housekeeper or a potential therapeutic target? Trends Pharmacol Sci 35: 178-186, 2014.

22. Zhou H, Huang T, Xiong Y, Peng L, Wang R and Zhang GJ: The prognostic value of proliferating cell nuclear antigen expression in colorectal cancer: A meta-analysis. Medicine (Baltimore) 97: e13752, 2018

23. Hu L, Li HL, Li WF, Chen JM, Yang JT, Gu JJ and Xin L: Clinical significance of expression of proliferating cell nuclear antigen and E-cadherin in gastric carcinoma. World J Gastroenterol 23 3721-3729, 2017.
24. Stenner M, Demgensky A, Molls C, Hardt A, Luers JC, Grosheva M, Huebbers CU and Klussmann JP: Prognostic value of proliferating cell nuclear antigen in parotid gland cancer. Eur Arch Otorhinolaryngol 269: 1225-1232, 2012

25. Kinugasa S, Tachibana M, Hishikawa Y, Abe S, Yoshimura H, Monden N, Dhar DK and Nagasue N: Prognostic significance of proliferating cell nuclear antigen (PCNA) in squamous cell carcinoma of the esophagus. Jpn J Clin Oncol 26: 405-410, 1996.

26. Kimos MC, Wang S, Borkowski A, Yang GY, Yang CS, Perry K, Olaru A, Deacu E, Sterian A, Cottrell J, et al: Esophagin and proliferating cell nuclear antigen (PCNA) are biomarkers of human esophageal neoplastic progression. Int J Cancer 111: 415-417, 2004.

27. Al-Ghamdi S, Albasri A, Cachat J, Ibrahem S, Muhammad BA, Jackson D, Nateri AS, Kindle KB and Ilyas M: Cten is targeted by kras signalling to regulate cell motility in the colon and pancreas. PLoS One 6: e20919, 2011.

28. Chan LK, Chiu YT, Sze KM and Ng IO: Tensin4 is up-regulated by EGF-induced ERK1/2 activity and promotes cell proliferation and migration in hepatocellular carcinoma. Oncotarget 6 : 20964-20976, 2015.

29. Al-Ghamdi S, Cachat J, Albasri A, Ahmed M, Jackson D, Zaitoun A, Guppy N, Otto WR, Alison MR, Kindle KB and Ilyas M: C-terminal tensin-like gene functions as an oncogene and promotes cell motility in pancreatic cancer. Pancreas 42 : 135-140, 2013.

30. Liao YC, Chen NT, Shih YP, Dong Y and Lo SH: Up-regulation of C-terminal tensin-like molecule promotes the tumorigenicity of colon cancer through beta-catenin. Cancer Res 69: 4563-4566, 2009.

31. Martuszewska D, Ljungberg B, Johansson M, Landberg G, Oslakovic C, Dahlbäck B and Hafizi S: Tensin3 is a negative regulator of cell migration and all four tensin family members are downregulated in human kidney cancer. PLoS One 4: e4350, 2009.

32. Wu WM and Liao YC: Downregulation of C-terminal tensin-like protein (CTEN) suppresses prostate cell proliferation and contributes to acinar morphogenesis. Int J Mol Sci 19: E3190, 2018.

33. Nie F, Yu X, Huang M, Wang Y, Xie M, Ma H, Wang Z, De W and Sun M: Long noncoding RNA ZFAS1 promotes gastric cancer cells proliferation by epigenetically repressing KLF2 and NKD2 expression. Oncotarget 8: 38227-38238, 2017.

34. Wang W and Xing C: Upregulation of long noncoding RNA ZFAS1 predicts poor prognosis and prompts invasion and metastasis in colorectal cancer. Pathol Res Pract 212: 690-695, 2016.

35. Li T, Xie J, Shen C, Cheng D, Shi Y, Wu Z, Deng X, Chen H, Shen B, Peng C, et al: Amplification of long noncoding RNA ZFAS1 promotes metastasis in hepatocellular carcinoma. Cancer Res 75: 3181-3191, 2015.

This work is licensed under a Creative Commons Attribution-NonCommercial-NoDerivatives 4.0 International (CC BY-NC-ND 4.0) License. 